\title{
MALT90: tracing the chemistry and kinematics of molecular clumps within the central molecular zone
}

\author{
Y. Contreras ${ }^{1}$, J. Rathborne ${ }^{1}$, J. Jackson ${ }^{2}$, J. Foster ${ }^{3}$, S. Longmore ${ }^{4}$ \\ and the MALT90 team \\ ${ }^{1}$ CSIRO Astronomy and Space Science \\ PO Box 76, Epping 2122, Sydney, Australia \\ email: yanett.contreras@csiro.au \\ ${ }^{2}$ Institute for Astrophysical Research, Boston University \\ Boston, MA 02215, USA \\ ${ }^{3}$ Yale Center for Astronomy and Astrophysics, Yale University \\ New Haven, CT 06520, USA \\ ${ }^{4}$ Astrophysics Research Institute, Liverpool John Moore University \\ Twelve Quays House, Egerton Wharf, Birkenhead CH 41 1LD,UK
}

\begin{abstract}
The MALT90 survey targets more than 2000 high-mass star-forming clumps in the Galactic plane obtaining small maps around each of them, in 16 molecular lines at $90 \mathrm{GHz}$. By observing several thousand high-mass star forming clumps MALT90 aims to characterize their global chemical and physical evolution. Here we summarize the survey parameters and show examples of the MALT90 data toward three clumps in the central molecular zone.
\end{abstract}

Keywords. stars: formation - ISM: clouds — radio lines: general

\section{Overview}

The Millimetre Astronomy Legacy Team $90 \mathrm{GHz}$ (MALT90) Survey (Foster et al. 2011, Jackson et al. 2013, Rathborne et al. 2013) recently obtained small maps toward 2000 high-mass star forming clumps identified from the ATLASGAL compact source catalog (Contreras et al. 2013). The survey was conducted using the $22 \mathrm{~m}$ Mopra telescope located in Australia, observing at 16 molecular lines simultaneously. The combination of molecular transitions covering a broad range in critical densities and excitation energies, and the key transitions that trace complex chemistry and shocked gas, reveal a wealth of information toward each clump. The survey maps are 3'x3', with a sensitivity of $0.2 \mathrm{~K} \mathrm{channel}^{-1}$, they have an angular resolution of 38 " and spectral resolution of $0.1 \mathrm{~km} \mathrm{~s}^{-1}$. The ATLASGAL clumps were selected to host the early stages of high-mass star-formation and to span the complete range of their evolutionary states (from prestellar clumps, to proto-stellar clumps, and on to HiI regions). The survey covered 450 clumps located in the central molecular zone (CMZ) (Figure 1). The combination of the data provided by MALT90 is key to understanding the properties of these clumps and can be used, for example, as a tool to understand the star formation in galaxies at high redshift (Kruijssen et al. 2013). All data products (reduced cubes and moment maps) are publicly available via ATOA $†$.

$\dagger$ http://atoa.atnf.csiro.au 


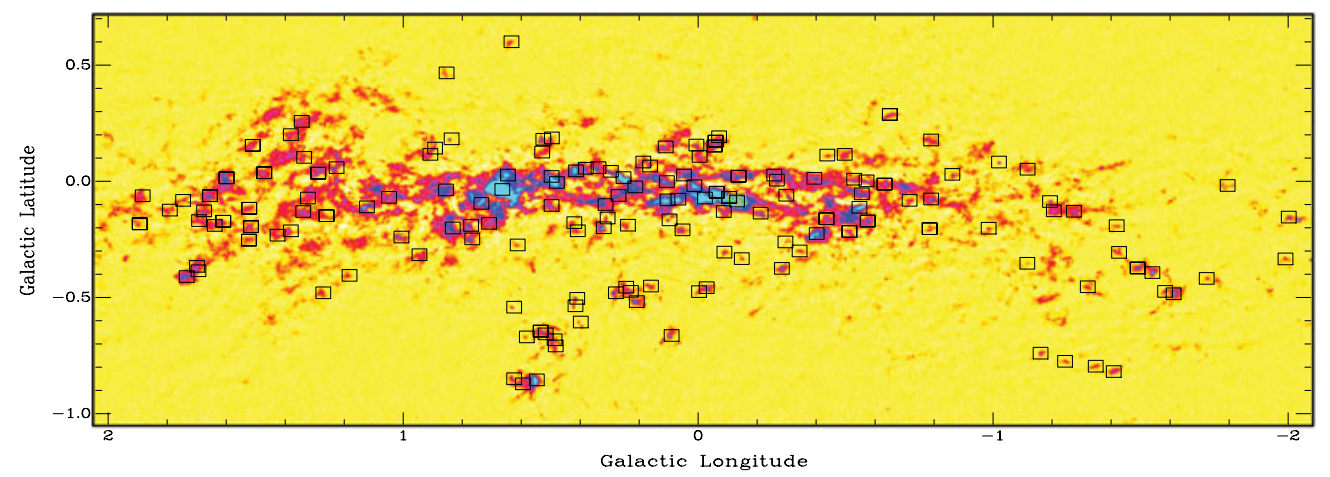

Figure 1. ATLASGAL $870 \mu \mathrm{m}$ continuum image toward the CMZ. The black boxes delineate the positions and size of the maps carried out as part of the MALT90 survey. [A COLOR VERsion is aVAilable online.]
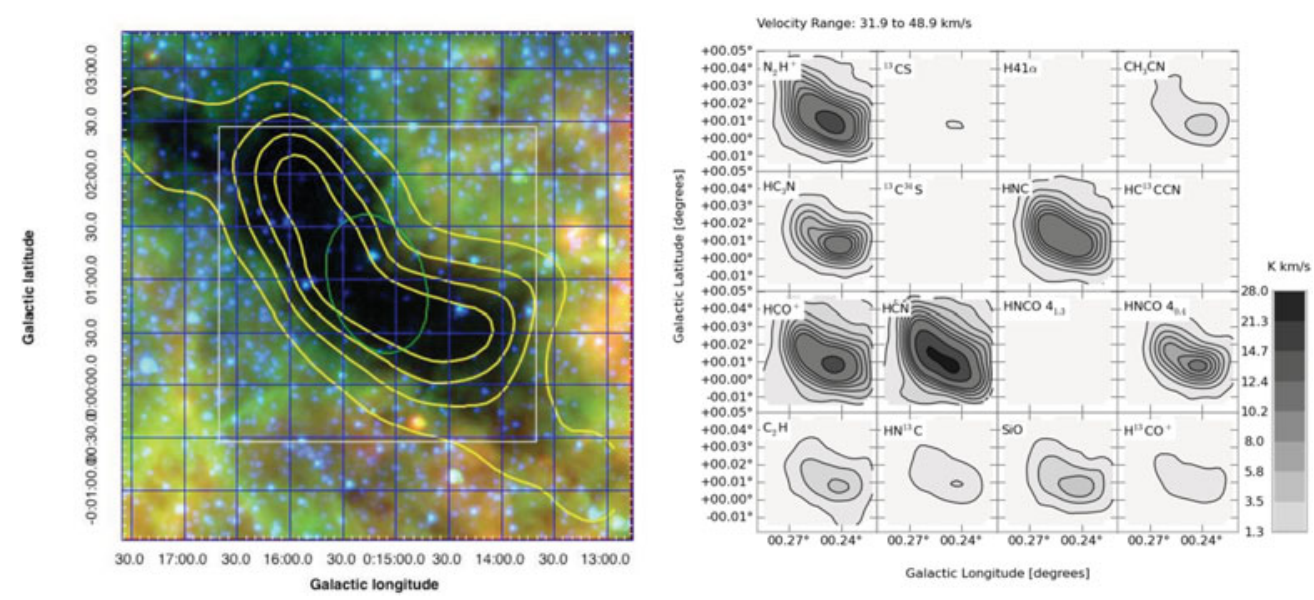

Figure 2. The Brick. Left: Color image of Spitzer data (red $24 \mu \mathrm{m}$, green $8 \mu \mathrm{m}$, blue 4.5 $\mu \mathrm{m})$ overlaid with ATLASGAL continuum data, where the white box shows the MALT90 observations. Right: Integrated intensity maps of the MALT90 emission toward the cloud. [A COLOR VERSION IS AVAILABLE ONLINE.]

\section{The Brick}

G000.253+00.016, "The Brick", is an extreme molecular clump located near the Galactic center, which is a potential candidate of an Arches-like massive cluster in an early stage of formation (Longmore et al. 2012). G000.253+00.016 appears dark in the mid- to far-IR, implying a low dust temperature and a high density, the presence of widespread emission from hot gas tracers suggests that the gas temperature may be higher $(\sim 70$ $\mathrm{K})$ than its dust temperature $(\sim 20 \mathrm{~K})$. The MALT90 data show that the gas has substructure with complex kinematics and broad line widths (Figure 2). We speculate that the observed morphology and kinematics of the molecular line emission traces the gas within a single externally heated collapsing clump. The detailed study of this clump will provide important information about the earliest stages of cluster formation (Rathborne et al. 2013). 


\section{References}

Contreras, Y., Schuller, F., Urquhart, J. S. et al. 2013, A\&A 549, 45

Foster, J. B., Jackson, J. M., Barris et al. 2011, ApJS 197, 25F

Jackson, J. M., Rathborne, J. M., Foster, J. B. et al. 2013, arXiv: 1310.1131, accepted to PASA

Kruijssen, J. M. Diederik, \& Longmore, Steven N. 2013, MNRAS 435, 2598

Longmore, Steven N., Rathborne, Jill, Bastian, Nate, et al. 2012, ApJ 746, 117

Rathborne, J. et al. 2013, Submitted 